\title{
A New Type of $\xi$-Open Sets Based on Operations
}

\author{
Haji M. Hasan \\ College of Basic Education \\ University of Duhok
}

Received on: 22/12/2011

Accepted on: 15/02/2012

\begin{abstract}
The aim of this paper is to introduce a new type of $\xi$-open sets in topological spaces which is called $\xi_{\gamma}$-open sets and we study some of their basic properties and characteristics.

Keywords: Open sets, $\xi$-Space.

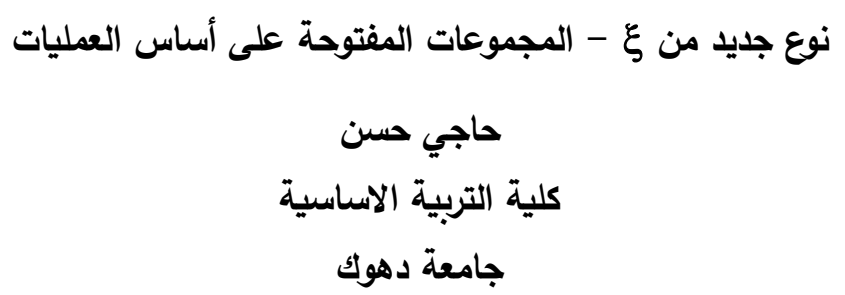

2012/02/15 : تاريخ القبول

تاريخ الاستلام: 2011/12/22

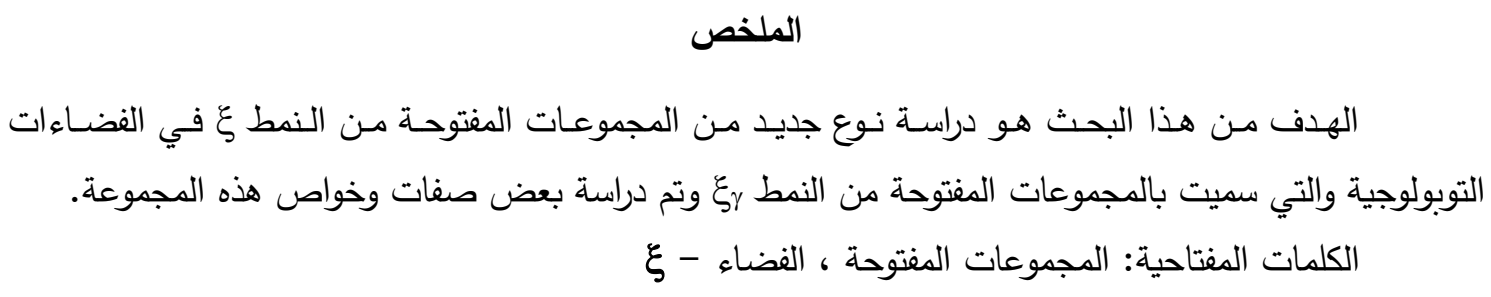

\section{Introduction}

Ogata [9], introduced the concept of an operation on a topology, then after authors defined some other types of sets such as $\gamma$-open [9], $\gamma$-semi-open [6], $\gamma$-pre semi-open [6] and $\gamma$ - $\beta$-open [1] sets in a topological space by using operations. In [4] the concept of $\xi$-open set in a topological space is introduced and studied.

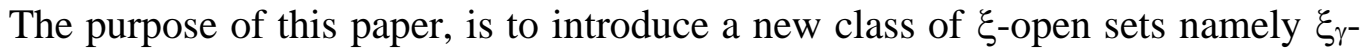
open sets and establish basic properties and relationships with other types of sets, also we define the notions of $\xi_{\gamma}$-neighbourhood, $\xi_{\gamma}$-derived, $\xi_{\gamma}$-closure and $\xi_{\gamma}$-interior of a set and give some of their properties which are mostly analogous to those properties of open sets. Throughout this paper, $(X, \tau)$ or(briefly, $X)$ mean a topological space on which no separation axioms are assumed unless explicitly stated. Let $\mathrm{A}$ be a subset of a topological space $\mathrm{X}, \mathrm{Cl}(\mathrm{A})$ and $\operatorname{Int}(\mathrm{A})$ are denoted respectively the closure and interior of A.

\section{Preliminaries.}

We start this section by introducing some definitions and results concerning sets and spaces which will be used later.

Definition 2.1. A subset A of a space $(X, \tau)$ is called: 
1) semi-open [7], if $\mathrm{A} \subseteq \mathrm{Cl}(\operatorname{Int}(\mathrm{A}))$.

2) regular open [2], if $\mathrm{A}=\operatorname{Int}(\mathrm{Cl}(\mathrm{A}))$.

The complement of semi-open (resp., regular open, preopen and $\alpha$-open) set is said to be semi-closed (resp., regular closed, preclosed and $\alpha$-closed ).

Definition 2.2. [4] An open subset $U$ of a space $X$ is called $\xi$-open if for each $x \in U$, there exists a semi-closed set $\mathrm{F}$ such that $\mathrm{x} \in \mathrm{F} \subseteq \mathrm{U}$. The family of all $\xi$-open subsets of a topological space $(X, \tau)$ is denoted by $\xi \mathrm{O}(\mathrm{X}, \tau)$ or (briefly $\xi \mathrm{O}(\mathrm{X})$ ). The complement of each $\xi$-open set is called $\xi$-closed set. The family of all $\xi$-closed subsets of a topological space $(\mathrm{X}, \tau)$ is denoted by $\xi \mathrm{C}(\mathrm{X}, \tau)$ or (briefly $\xi \mathrm{C}(\mathrm{X})$ ).

Definition 2.3. [5] Let $(X, \tau)$ be a topological space. An operation $\gamma$ on the topology $\tau$ is a mapping from $\tau$ into power set $\mathrm{P}(\mathrm{X})$ such that $\mathrm{V} \subseteq \gamma(\mathrm{V})$ for each $\mathrm{V} \in \tau$, where $\gamma(\mathrm{V})$ denotes the value of $\gamma$ at $\mathrm{V}$.

Definition 2.4. [8]

1) A subset $A$ of a topological space $(X, \tau)$ is called $\gamma$-open set if for each $x \in A$ there exists an open set $\mathrm{U}$ such that $\mathrm{X} \in \mathrm{U}$ and $\gamma(\mathrm{U}) \subseteq \mathrm{A}$. Clearly $\tau_{\gamma} \subseteq \tau$.

Complements of $\gamma$-open sets are called $\gamma$-closed.

2) The point $x \in X$ is in the $\gamma$-closure of a set $A \subseteq X$, if $\gamma(U) \cap A \neq \phi$, for each open set $\mathrm{U}$ containing $\mathrm{x}$. The $\gamma$-closure of a set $\mathrm{A}$ is denoted by $\mathrm{Cl}_{\gamma}(\mathrm{A})$.

3) Let $(X, \tau)$ be a topological space and A be subset of $X$, then $\tau_{\gamma}-\mathrm{Cl}(\mathrm{A})=\cap\{\mathrm{F}$ : $\mathrm{A} \subseteq$ $\left.\mathrm{F}, \mathrm{X} \backslash \mathrm{F} \in \tau_{\gamma}\right\}$.

Definition 2.5. [11] Let $(X, \tau)$ be a topological space and $A$ be subset of $X$, then $\tau_{\gamma^{-}}$ $\operatorname{Int}(\mathrm{A})=\cup\{\mathrm{U}: \mathrm{U}$ is $\gamma$-open set and $\mathrm{U} \subseteq \mathrm{A}\}$.

Definition 2.6. [1] Let $(X, \tau)$ be a topological space with an operation $\gamma$ on $\tau$ :

1) The $\gamma$-derived set of $A$ is defined by $\{x$ : for every $\gamma$-open set $U$ containing $x, U$ $\cap(\mathrm{A} \backslash\{\mathrm{x}\}) \neq \phi\}$

2) The $\gamma$-boundary of $\mathrm{A}$ is defined as $\tau_{\gamma}-\mathrm{Cl}(\mathrm{A}) \cap \tau_{\gamma}-\mathrm{Cl}(\mathrm{X} \backslash \mathrm{A})$.

Definition 2.7. [4] Let $(X, \tau)$ be a topological space and $A \subseteq X$, then:

1) $\xi$-interior of $\mathrm{A}$ is the union of all $\xi$-open sets contained in $\mathrm{A}$.

2) $\xi$-closure of $\mathrm{A}$ is the intersection of all $\xi$-closed sets containing $\mathrm{A}$.

Lemma 2.8. [4]

1) Let $\left(Y, \tau_{Y}\right)$ be a subspace of $(X, \tau)$. If $F \in S C(X, \tau)$ and $F \subseteq Y$, then $F \in S C\left(Y, \tau_{Y}\right)$.

2) Let $\left(Y, \tau_{Y}\right)$ be a subspace of $(X, \tau)$. If $F \in \operatorname{SC}\left(Y, \tau_{Y}\right)$ and $Y \in \operatorname{SC}(X, \tau)$, then $F \in$ $\mathrm{SC}(\mathrm{X}, \tau)$.

Lemma 2.9 [4]

1) Let $Y$ be a regular open subspace of a space $X$. If $G \in \xi O(Y)$, then $G \in \xi O(X)$.

2) Let $Y$ be a subspace of a space $X$ and $Y \in S C(X)$. If $G \in \xi O(X)$ and $G \subseteq Y$, then $G$ $\in \xi \mathrm{O}(\mathrm{Y})$.

\section{3. $\xi_{\gamma}$-Open Sets}

In this section, a new class of $\xi$-open sets called $\xi_{\gamma}$-open sets in topological spaces is introduced. We define $\gamma$ to be a mapping on $\xi \mathrm{O}(\mathrm{X})$ into $\mathrm{P}(\mathrm{X})$ and we say that $\gamma: \xi \mathrm{O}(\mathrm{X}) \rightarrow \mathrm{P}(\mathrm{X})$ is an $\xi$-operation on $\xi \mathrm{O}(\mathrm{X})$ if $\mathrm{V} \subseteq \gamma(\mathrm{V})$, for each $\mathrm{V} \in \xi \mathrm{O}(\mathrm{X})$. 
Definition 3.1 A subset A of a space $X$ is called $\xi_{\gamma}$-open if for each point $\mathrm{x} \in \mathrm{A}$, there exist an $\xi$-open set $\mathrm{U}$ such that $\mathrm{x} \in \mathrm{U} \subseteq \gamma(\mathrm{U}) \subseteq \mathrm{A}$.

The family of all $\xi_{\gamma}$-open subset of a topological space $(X, \tau)$ is denoted by $\xi_{\gamma} \mathrm{O}(\mathrm{X}, \tau)$ or (briefly $\xi_{\gamma} \mathrm{O}(\mathrm{X})$ ).

A subset $B$ of a space $X$ is called $\xi_{\gamma}$-closed if $X \backslash B$ is $\xi_{\gamma}$-open. The family of all $\xi_{\gamma}$-closed subsets of a topological space $(\mathrm{X}, \tau)$ is denoted by $\xi_{\gamma} \mathrm{C}(\mathrm{X}, \tau)$ or (briefly $\left.\xi_{\gamma} \mathrm{C}(\mathrm{X})\right)$.

Remark 3.2 From the definition of the operation $\gamma$, it is clear that $\gamma(X)=X$ for any $\xi$ operation $\gamma$. For competence, it is assumed that $\gamma(\phi)=\phi$ for any $\xi$-operation $\gamma$.

Remark 3.3 It is clear from the definition that every $\xi_{\gamma}$-open subset of a space $\mathrm{X}$ is $\xi$ open, but the converse is not true in general as shown in the following example:

Example 3.5. Consider $X=\{a, b, c, d\}$ with the topology $\tau=\{\phi, X,\{c\},\{a, b\},\{a, b, c\}\}$. Define an $\xi$-operation $\gamma$ by

$$
\gamma(A)= \begin{cases}A & \text { if } \quad a \in A \\ X & \text { if } a \notin A\end{cases}
$$

Then $\{\mathrm{c}\}$ is open and $\xi$-open but $\{\mathrm{c}\} \notin \xi_{\gamma} \mathrm{O}(\mathrm{X})$.

Proposition 3.6. Every $\xi_{\gamma}$-open set of a space $\mathrm{X}$ is $\gamma$-open.

Proof. Let $\mathrm{A}$ be $\xi_{\gamma}$-open in a topological space $(\mathrm{X}, \tau)$, then for each point $\mathrm{x} \in \mathrm{A}$, there exists an $\xi$-open set $\mathrm{U}$ such that $\mathrm{x} \in \mathrm{U} \subseteq \gamma(\mathrm{U}) \subseteq \mathrm{A}$. Since every $\xi$-open set is open, this implies that $\mathrm{A}$ is a $\gamma$-open set.

The following example shows that the converse of the above proposition is not true in general.

Example 3.7 Consider $X=\{a, b, c\}$ with topology $\tau=\{\phi, X,\{a\}\}$. Define an $\xi$ operation $\gamma$ by $\gamma(\mathrm{A})=\mathrm{A}$, for any subset $\mathrm{A}$ of $\mathrm{X}$. Then, $\{\mathrm{a}\}$ is $\gamma$-open set but not $\xi$-open set. Hence, it is not $\xi_{\gamma}$-open.

The following result shows that any union of $\xi_{\gamma}$-open sets in a topological space $(\mathrm{X}, \tau)$ is $\xi_{\gamma}$-open.

Proposition 3.8 Let $\left\{A_{\lambda}\right\}_{\lambda \in \Delta}$ be a collection of $\xi_{\gamma}$-open sets in a topological space (X, $\tau)$. Then, $\cup_{\lambda \in \Delta} A_{\lambda}$ is $\xi_{\gamma \text {-open. }}$

Proof. Let $\mathrm{x} \in \bigcup_{\lambda \in \Delta} A_{\lambda}$, then $\mathrm{x} \in A_{\lambda}$ for some $\lambda \in \Delta$. Since, $A_{\lambda}$ is an $\xi_{\gamma}$-open set, then there exists an $\xi_{\gamma}$-open set $\mathrm{U}$ containing $\mathrm{x}$ and $\gamma(\mathrm{U}) \subseteq A_{\lambda} \subseteq \cup_{\lambda \in \Delta} A_{\lambda}$. Therefore, $\cup_{\lambda \in \Delta} A_{\lambda}$ is an $\xi_{\gamma}$-open set in a topological space $(\mathrm{X}, \tau)$.

The following example shows that the intersection of two $\xi_{\gamma}$-open sets need not be an $\xi_{\gamma}$-open set.

Example 3.9 Consider $X=\{a, b, c\}$ with discrete topology on $X$. Define an $\xi$-operation $\gamma$ by 


$$
\gamma(A)= \begin{cases}\{a, b\} & \text { if } A=\{a\} \text { or }\{b\} \\ A & \text { otherwise }\end{cases}
$$

Let $A=\{a, b\}$ and $B=\{b, c\}$, it is clear that $A$ and $B$ are $\xi_{\gamma}$-open sets, but $A \cap B=\{b\}$ is not $\xi_{\gamma}$-open set.

From the above example, we notice that the family of all $\xi_{\gamma}$-open subsets of a space $\mathrm{X}$ is a supratopology and need not be a topology in general.

Proposition 3.10 The set $A$ is $\xi_{\gamma}$-open in the space $(X, \tau)$ if and only if for each $\mathrm{x} \in \mathrm{A}$, there exists an $\xi$-open set $\mathrm{B}$ such that $\mathrm{x} \in \mathrm{B} \subseteq \mathrm{A}$.

Proof. Suppose that $A$ is an $\xi_{\gamma}$-open set in the space $(X, \tau)$. Then, for each $\mathrm{x} \in \mathrm{A}$, put $\mathrm{B}=\mathrm{A}$ is an $\xi$-open set such that $\mathrm{x} \in \mathrm{B} \subseteq \mathrm{A}$.

Conversely, suppose that for each $\mathrm{x} \in \mathrm{A}$, there exists an $\xi$-open set $\mathrm{B}_{\mathrm{x}}$ such that $\mathrm{x} \in \mathrm{B}_{\mathrm{x}}$ $\subseteq \mathrm{A}$, thus $\mathrm{A}=\cup \mathrm{B}_{\mathrm{x}}$ where $\mathrm{B}_{\mathrm{x}} \in \xi_{\gamma} \mathrm{O}(\mathrm{X})$ for each $\mathrm{x} \in \mathrm{A}$. Therefore, $\mathrm{A}$ is $\xi_{\gamma}$-open set.

Definition 3.11 Let $(X, \tau)$ be a topological space. A mapping $\gamma: \xi O(X) \rightarrow P(X)$ is said to be :

1) $\xi$-identity on $\xi \mathrm{O}(\mathrm{X})$ if $\gamma(\mathrm{A})=\mathrm{A}$ for all $\mathrm{A} \in \xi \mathrm{O}(\mathrm{X})$.

2) $\xi$-monotone on $\xi \mathrm{O}(\mathrm{X})$ if for all $\mathrm{A}, \mathrm{B} \in \xi \mathrm{O}(\mathrm{X}), \mathrm{A} \subseteq \mathrm{B}$ implies $\gamma(\mathrm{A}) \subseteq \gamma(\mathrm{B})$.

3) $\xi$-idempotent on $\xi \mathrm{O}(\mathrm{X})$ if $\gamma(\gamma(\mathrm{A}))=\gamma(\mathrm{A})$ for all $\mathrm{A} \in \xi \mathrm{O}(\mathrm{X})$.

4) $\xi$-additive on $\xi \mathrm{O}(\mathrm{X})$ if $\gamma(\mathrm{A} \cup \mathrm{B})=\gamma(\mathrm{A}) \cup \gamma(\mathrm{B})$ for all $\mathrm{A}, \mathrm{B} \in \xi \mathrm{O}(\mathrm{X})$.

If $\cup_{i \in I} \gamma\left(A_{i}\right) \subseteq \gamma\left(\cup_{i \in I} A_{i}\right)$ for any collection $\left\{A_{i}\right\}_{i \in I} \subseteq \xi O(X)$, then $\gamma$ is said to be $\xi$ subadditive on $\xi \mathrm{O}(\mathrm{X})$.

Proposition 3.12. Let $\gamma$ be an $\xi$-operation. Then, $\gamma$ is $\xi$-monotone on $\xi \mathrm{O}(\mathrm{X})$ if and only if $\gamma$ is subadditive on $\xi \mathrm{O}(\mathrm{X})$.

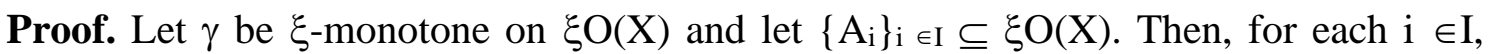
$\gamma\left(\mathrm{A}_{\mathrm{i}}\right) \subseteq \gamma\left(\cup_{\mathrm{i} \in \mathrm{I}} \mathrm{A}_{\mathrm{i}}\right)$ and thus $\cup_{\mathrm{i} \in \mathrm{I}} \gamma\left(\mathrm{A}_{\mathrm{i}}\right) \subseteq \gamma\left(\cup_{\mathrm{i} \in \mathrm{I}} \mathrm{A}_{\mathrm{i}}\right)$. Therefore, $\gamma$ is $\xi$ - subadditive on $\xi \mathrm{O}(\mathrm{X})$.

Conversely, if $\gamma$ is subadditive on $\xi \mathrm{O}(\mathrm{X})$, and $\mathrm{A}, \mathrm{B} \in \xi \mathrm{O}(\mathrm{X})$ with $\mathrm{A} \subseteq \mathrm{B}$, then $\gamma(\mathrm{A}) \subseteq$ $\gamma(\mathrm{A}) \cup \gamma(\mathrm{B}) \subseteq \gamma(\mathrm{A} \cup \mathrm{B})=\gamma(\mathrm{B})$. Thus, $\gamma$ is $\xi$-monotone on $\xi \mathrm{O}(\mathrm{X})$.

The following result shows that if $\gamma$ is $\xi$-monotone, then the family of $\xi_{\gamma}$-open sets is a topology on X.

Proposition 3.13 If $\gamma$ is $\xi$-monotone, then the family of $\xi_{\gamma}$-open sets is a topology on $\mathrm{X}$.

Proof. Clearly $\phi, X \in \xi_{\gamma} \mathrm{O}(\mathrm{X})$ and by Proposition3.8, the union of any family $\xi_{\gamma}$-open sets is $\xi_{\gamma}$-open set. To complete the proof, it is enough to show that the finite intersection of $\xi_{\gamma}$-open sets is an $\xi_{\gamma}$-open set. Let $\mathrm{A}$ and $\mathrm{B}$ be two $\xi_{\gamma}$-open sets and let $\mathrm{x}$ $\in \mathrm{A} \cap \mathrm{B}$, then $\mathrm{x} \in \mathrm{A}$ and $\mathrm{x} \in \mathrm{B}$, so there exists $\xi_{\gamma}$-open sets namely $\mathrm{U}$ and $\mathrm{V}$ such that $\mathrm{x} \in \mathrm{U} \subseteq \gamma(\mathrm{U}) \subseteq \mathrm{A}$ and $\mathrm{x} \in \mathrm{V} \subseteq \gamma(\mathrm{V}) \subseteq \mathrm{B}$, since $\mathrm{U}$ and $\mathrm{V}$ are $\xi$-open sets then $\mathrm{U} \cap \mathrm{V}$ is $\xi$-open, but $\mathrm{U} \cap \mathrm{V} \subseteq \mathrm{U}$ and $\mathrm{U} \cap \mathrm{V} \subseteq \mathrm{V}$, but $\gamma$ is $\xi$-monotone operation, therefore $\gamma(\mathrm{U}$ $\cap \mathrm{V}) \subseteq \gamma(\mathrm{U}) \cap \gamma(\mathrm{V}) \subseteq \mathrm{A} \cap \mathrm{B}$. Thus, $\mathrm{A} \cap \mathrm{B}$ is an $\xi_{\gamma}$-open set. This completes the proof.

Proposition 3.14 Let $\mathrm{Y}$ be a semi-closed subspace of a space $\mathrm{X}$. If $\mathrm{A} \in \xi_{\gamma} \mathrm{O}(\mathrm{X}, \tau)$ and $\mathrm{A}$ $\subseteq \mathrm{Y}$, then $\mathrm{A} \in \xi_{\gamma} \mathrm{O}\left(\mathrm{Y}, \tau_{\mathrm{Y}}\right)$, where $\gamma$ is $\xi$-identity on $\xi \mathrm{O}(\mathrm{Y})$. 
Proof. Let $\mathrm{A} \in \xi_{\gamma} \mathrm{O}(\mathrm{X}, \tau)$, then $\mathrm{A} \in \xi \mathrm{O}(\mathrm{X}, \tau)$ and for each $\mathrm{x} \in \mathrm{A}$ there exists an $\xi$-open set $\mathrm{U}$ in $\mathrm{X}$ such that $\mathrm{x} \in \mathrm{U} \subseteq \gamma(\mathrm{U}) \subseteq \mathrm{A}$. Since, $\mathrm{A} \in \xi \mathrm{O}(\mathrm{X}, \tau)$ and $\mathrm{A} \subseteq \mathrm{Y}$, where $\mathrm{Y}$ is semi-closed in $\mathrm{X}$, then by Proposition 2.14, $\mathrm{U} \in \xi_{\gamma} / \mathrm{O}\left(\mathrm{Y}, \tau_{\mathrm{Y}}\right)$. Hence, $\mathrm{A} \in \xi_{\gamma} / \mathrm{O}\left(\mathrm{Y}, \tau_{\mathrm{Y}}\right)$.

Proposition 3.15 Let $\mathrm{Y}$ be a regular open subspace of a space $(\mathrm{X}, \tau)$ and $\gamma$ is an $\xi$ identity on $\xi \mathrm{O}(\mathrm{X})$. If $\mathrm{A} \in \xi_{\gamma} \mathrm{O}\left(\mathrm{Y}, \tau_{\mathrm{Y}}\right)$ and $\mathrm{Y} \in \xi \mathrm{O}(\mathrm{X}, \tau)$, then $\mathrm{A} \in \xi_{\gamma} \mathrm{O}(\mathrm{X}, \tau)$.

Proof. Let $\mathrm{A} \in \xi_{\gamma} \mathrm{O}\left(\mathrm{Y}, \tau_{\mathrm{Y}}\right)$, then $\mathrm{A} \in \xi \mathrm{O}\left(\mathrm{Y}, \tau_{\mathrm{Y}}\right)$ and for each $\mathrm{x} \in \mathrm{A}$ there exists an $\xi$ open set $\mathrm{U}$ in $\mathrm{Y}$ such that $\mathrm{x} \in \mathrm{U} \subseteq \gamma /(\mathrm{U}) \subseteq \mathrm{A}$. Since, $\mathrm{Y} \in \xi \mathrm{O}(\mathrm{X}, \tau)$ and $\mathrm{A} \in \xi \mathrm{O}(\mathrm{Y}$, $\tau_{\mathrm{Y}}$, then by Proposition 2.13, $\mathrm{U} \in \xi \mathrm{O}(\mathrm{X}, \tau)$. Hence, $\mathrm{A} \in \xi_{\gamma} \mathrm{O}(\mathrm{X}, \tau)$.

\section{Other Properties of $\xi_{\gamma}$-Open Sets}

In this section, we define and study some properties of $\xi_{\gamma}$-neighbourhood of a

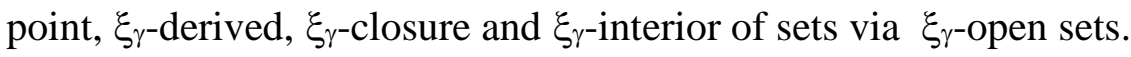

Definition 4.1 Let $(X, \tau)$ be a topological space and $x \in X$, then a subset $N$ of $X$ is said to be $\xi_{\gamma}$-neighbourhood of $\mathrm{x}$, if there exists an $\xi_{\gamma}$-open set $\mathrm{U}$ in $\mathrm{X}$ such that $\mathrm{x} \in \mathrm{U} \subseteq \mathrm{N}$.

Proposition 4.2 Let $(X, \tau)$ be a topological space. A subset $A$ of $X$ is $\xi_{\gamma}$-open if and only if it is an $\xi_{\gamma}$-neighbourhood of each its points.

Proof. Let $A \subseteq X$ be an $\xi_{\gamma}$-open set. Since, for every $\mathrm{x} \in \mathrm{A}, \mathrm{x} \in \mathrm{A} \subseteq \mathrm{A}$ and $\mathrm{A}$ is $\xi_{\gamma^{-}}$ open, then $\mathrm{A}$ is an $\xi_{\gamma}$-neighbourhood of each its points.

Conversely, suppose that $\mathrm{A}$ is an $\xi_{\gamma}$-neighbourhood of each its points. Then, for each $\mathrm{x}$ $\in A$, there exists $B_{x} \in \xi_{\gamma} O(X)$ such that $B_{x} \subseteq A$. Then, $A=\cup\left\{B_{x}: x \in A\right\}$. Since, each $\mathrm{B}_{\mathrm{x}}$ is $\xi_{\gamma}$-open, It follows that $\mathrm{A}$ is an $\xi_{\gamma}$-open set.

Definition 4.3 Let $(X, \tau)$ be a topological space with an operation $\gamma$ on $\xi \mathrm{O}(\mathrm{X})$. A point $\mathrm{X}$ $\in \mathrm{X}$ is said to be $\xi_{\gamma}$-limit point of a set $\mathrm{A}$ if for each $\xi_{\gamma}$-open set $\mathrm{U}$ containing $\mathrm{X}$, then $\mathrm{U}$ $\cap(\mathrm{A} \backslash\{\mathrm{x}\}) \neq \phi$. The set of all $\xi_{\gamma}$-limit points of $\mathrm{A}$ is called $\xi_{\gamma}$-derived set of $\mathrm{A}$ and denoted by $\xi_{\gamma}-\mathrm{D}(\mathrm{A})$.

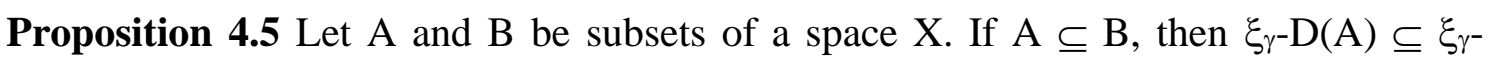
$\mathrm{D}(\mathrm{B})$.

Proof. Obvious.

Some properties of $\xi_{\gamma}$-derived sets are stated in the following proposition.

Proposition 4.6 Let A and B be any two subsets of a space $X$, and $\gamma$ be an operation on $\xi \mathrm{O}(\mathrm{X})$. Then, we have the following properties:

1) $\xi_{\gamma}-\mathrm{D}(\phi)=\phi$.

2) If $x \in \xi_{\gamma}-\mathrm{D}(\mathrm{A})$, then $\mathrm{x} \in \xi_{\gamma} \mathrm{D}(\mathrm{A} \backslash\{\mathrm{x}\})$.

3) $\xi_{\gamma}-\mathrm{D}(\mathrm{A}) \cup \xi_{\gamma}-\mathrm{D}(\mathrm{B}) \subseteq \xi_{\gamma}-\mathrm{D}(\mathrm{A} \cup \mathrm{B})$.

4) $\xi_{\gamma}-\mathrm{D}(\mathrm{A} \cap \mathrm{B}) \subseteq \xi_{\gamma}-\mathrm{D}(\mathrm{A}) \cap \xi_{\gamma}-\mathrm{D}(\mathrm{B})$.

5) $\xi_{\gamma}-\mathrm{D}\left(\xi_{\gamma}-\mathrm{D}(\mathrm{A})\right) \backslash \mathrm{A} \subseteq \xi_{\gamma}-\mathrm{D}(\mathrm{A})$.

6) $\xi_{\gamma-\mathrm{D}} \mathrm{D} \cup \xi_{\gamma-\mathrm{D}}(\mathrm{A}) \subseteq \mathrm{A} \cup \xi_{\gamma-\mathrm{D}}(\mathrm{A})$.

Proof. Straightforward.

In general, the equalities of (3), (4) and (6) of the above proposition do not hold, as is shown in the following examples. 
Example 4.7 Consider $X=\{a, b, c\}$ with discrete topology on $X$. Define an operation $\gamma$ on $\xi \mathrm{O}(\mathrm{X})$ by

$$
\gamma(A)=\left\{\begin{array}{lll}
A & \text { if } & A=\{b\} \text { or }\{a, b\} \text { or }\{a, c\} \\
X & \text { otherwise }
\end{array}\right.
$$

Now, if $A=\{a, b\}$ and $B=\{a, c\}$, then $\xi_{\gamma}-D(A)=\{c\}, \xi_{\gamma}-D(B)=\{c\}$ and $\xi_{\gamma}-D(A \cup B)$ $=\{\mathrm{a}, \mathrm{c}\}$, where $\mathrm{A} \cup \mathrm{B}=\mathrm{X}$, this implies that $\xi_{\gamma} \mathrm{D}(\mathrm{A}) \cup \xi_{\gamma}-\mathrm{D}(\mathrm{B}) \neq \xi_{\gamma}-\mathrm{D}(\mathrm{A} \cup \mathrm{B})$.

Example 4.8 Consider $X=\{a, b, c, d\}$ with the topology $\tau=\{\phi, X,\{a\},\{b\},\{c\},\{a$, $b\},\{a, c\},\{b, c\},\{a, b, c\}\}$. Define an operation $\gamma$ on $\xi O(X) b y$.

$$
\gamma(A)= \begin{cases}A & \text { if } b \in A \\ X & \text { if } b \notin A\end{cases}
$$

Now, if we let $A=\{a, b\}$ and $B=\{c, d\}$, then $\xi_{\gamma}-D(A)=\{a, c, d\}, \xi_{\gamma}-D(B)=\{d\}$, hence $\xi_{\gamma}-\mathrm{D}(\mathrm{A}) \cap \xi_{\gamma}-\mathrm{D}(\mathrm{B})=\{\mathrm{d}\}$, but $\xi_{\gamma}-\mathrm{D}(\mathrm{A} \cap \mathrm{B})=\phi$, where $\mathrm{A} \cap \mathrm{B}=\phi$, this implies that $\xi_{\gamma^{-}}$ $\mathrm{D}(\mathrm{A} \cap \mathrm{B}) \neq \xi_{\gamma}-\mathrm{D}(\mathrm{A}) \cap \xi_{\gamma} \mathrm{D}(\mathrm{B})$. Also $\xi \mathrm{D}(\mathrm{A})=\{\mathrm{d}\}$, therefore $\xi_{\gamma} \mathrm{D}(\mathrm{A}) \not \subset \xi \mathrm{D}(\mathrm{A})$.

Definition 4.9 Let $A$ be a subset of a topological space $(X, \tau)$ and $\gamma$ be an operation on $\xi \mathrm{O}(\mathrm{X})$. The intersection of all $\xi_{\gamma}$-closed sets containing $\mathrm{A}$ is called the $\xi_{\gamma}$-closure of A and denoted by $\xi_{\gamma}-\mathrm{Cl}(\mathrm{A})$.

Here, we introduce some properties of $\xi_{\gamma}$-closure of the sets.

Proposition 4.10 Let $(X, \tau)$ be a topological space and $\gamma$ be an operation on $\xi \mathrm{O}(\mathrm{X})$. For any subsets $\mathrm{A}$ and $\mathrm{B}$ of $\mathrm{X}$, we have the following:

1) $\mathrm{A} \subseteq \xi_{\gamma}-\mathrm{Cl}(\mathrm{A})$.

2) $\xi_{\gamma}-\mathrm{Cl}(\mathrm{A})$ is an $\xi_{\gamma}$-closed set in $\mathrm{X}$.

3) $\mathrm{A}$ is an $\xi_{\gamma}$-closed set if and only if $\mathrm{A}=\xi_{\gamma}-\mathrm{Cl}(\mathrm{A})$.

4) $\xi_{\gamma}-\mathrm{Cl}(\phi)=\phi$ and $\xi_{\gamma}-\mathrm{Cl}(\mathrm{X})=\mathrm{X}$.

5) $\xi_{\gamma-} \mathrm{Cl}(\mathrm{A}) \cup \xi_{\gamma-} \mathrm{Cl}(\mathrm{B}) \subseteq \xi_{\gamma}-\mathrm{Cl}(\mathrm{A} \cup \mathrm{B})$.

6) $\xi_{\gamma}-\mathrm{Cl}(\mathrm{A} \cap \mathrm{B}) \subseteq \xi_{\gamma} \mathrm{Cl}(\mathrm{A}) \cap \xi_{\gamma}-\mathrm{Cl}(\mathrm{B})$.

Proof. They are obvious.

In general, the equalities of (5) and (6) of the above proposition does not hold, as is shown in the following examples:

Example 4.11 Consider $X=\{a, b, c\}$ with discrete topology on $X$. Define an operation $\gamma$ on $\xi \mathrm{O}(\mathrm{X})$ by

$$
\gamma(A)=\left\{\begin{array}{lll}
A & \text { if } & A=\{a, b\} \text { or }\{a, c\} \\
X & \text { otherwise }
\end{array}\right.
$$

Then, $\xi_{\gamma} \mathrm{O}(\mathrm{X})=\{\phi, X,\{\mathrm{a}, \mathrm{b}\},\{\mathrm{a}, \mathrm{c}\}\}$. Now, if we let $\mathrm{A}=\{\mathrm{b}\}$ and $\mathrm{B}=\{\mathrm{c}\}$, then $\xi_{\gamma-}$ $\mathrm{CL}(\mathrm{A})=\mathrm{A}, \xi_{\gamma}-\mathrm{D}(\mathrm{B})=\mathrm{B}$ and $\xi_{\gamma} \mathrm{Cl}(\mathrm{A} \cup \mathrm{B})=\mathrm{X}$, where $\mathrm{A} \cup \mathrm{B}=\{\mathrm{b}, \mathrm{c}\}$, this implies that $\xi_{\gamma}-\mathrm{Cl}(\mathrm{A}) \cup \xi_{\gamma-} \mathrm{Cl}(\mathrm{B}) \neq \xi_{\gamma}-\mathrm{Cl}(\mathrm{A} \cup \mathrm{B})$.

Example 4.12 Consider $X=\{a, b, c, d\}$ with the topology $\tau=\{\phi, X,\{a\},\{b\},\{c\},\{a$, $b\},\{a, c\},\{b, c\},\{a, b, c\}\}$. Define an operation $\gamma$ on $\xi O(X) b y$. 


$$
\gamma(A)= \begin{cases}A & \text { if } \quad b \in A \\ X & \text { if } b \notin A\end{cases}
$$

It is clear that $\xi_{\gamma}-\mathrm{O}(\mathrm{X})=\{\phi, X,\{b\},\{a, b\},\{b, c\},\{a, b, c\}\}$. Now, if we let $A=\{c\}$ and $\mathrm{B}=\{\mathrm{d}\}$, then $\xi_{\gamma}-\mathrm{Cl}(\mathrm{A})=\{\mathrm{c}, \mathrm{d}\}$ and $\xi_{\gamma}-\mathrm{Cl}(\mathrm{B})=\{\mathrm{d}\}$,

hence $\xi_{\gamma}-\mathrm{Cl}(\mathrm{A}) \cap \xi_{\gamma}-\mathrm{Cl}(\mathrm{B})=\{\mathrm{d}\}$, but $\xi_{\gamma}-\mathrm{Cl}(\mathrm{A} \cap \mathrm{B})=\phi$, where $\mathrm{A} \cap \mathrm{B}=\phi$, this implies that $\xi_{\gamma}-\mathrm{CL}(\mathrm{A} \cap \mathrm{B}) \neq \xi_{\gamma}-\mathrm{Cl}(\mathrm{A}) \cap \xi_{\gamma} \mathrm{Cl}(\mathrm{B})$.

Now, if we let $A=\{b\}$, we see that $\xi \mathrm{Cl}(\mathrm{A})=\{\mathrm{b}, \mathrm{d}\}$, but $\xi_{\gamma}-\mathrm{Cl}(\mathrm{A})=\mathrm{X}$. Hence, $\xi_{\gamma}-\mathrm{Cl}(\mathrm{A})$ $\not \subset \xi \mathrm{Cl}(\mathrm{A})$.

Proposition 4.13 A subset A of a topological space $\mathrm{X}$ is an $\xi_{\gamma}$-closed set if and only if it contains the set of its $\xi_{\gamma}$-limit points.

Proof. Assume that A is an $\xi_{\gamma}$-closed set and if possible that $\mathrm{x}$ is an $\xi_{\gamma}$-limit point of A which belongs to $\mathrm{X} \backslash \mathrm{A}$, then $\mathrm{X} \backslash \mathrm{A}$ is an $\xi_{\gamma}$-open set containing the $\xi_{\gamma}$-limit point of $\mathrm{A}$, therefore, $\mathrm{A} \cap(\mathrm{X \backslash A}) \neq \phi$, which is contradiction.

Conversely, assume that $A$ is containing the set of its $\xi_{\gamma}$-limit points. For each $x \in X \backslash A$, there exists an $\xi_{\gamma}$-open set $\mathrm{U}$ containing $\mathrm{x}$ such that $\mathrm{A} \cap \mathrm{U}=\phi$, implies that $\mathrm{x} \in \mathrm{U} \subseteq$ $\mathrm{X} \backslash \mathrm{A}$, so by Proposition 3.10, $\mathrm{X} \backslash \mathrm{A}$ is an $\xi_{\gamma}$-open set hence, $\mathrm{A}$ is an $\xi_{\gamma}$-closed set.

Proposition 4.14 Let $A$ be a subset of a topological space $(X, \tau)$ and $\gamma$ be an $\xi$ operation. Then, $\mathrm{x} \in \xi_{\gamma} \mathrm{Cl}(\mathrm{A})$ if and only if for every $\xi_{\gamma}$-open set $\mathrm{V}$ of $\mathrm{X}$ containing $\mathrm{x}, \mathrm{A}$ $\cap \mathrm{V} \neq \phi$.

Proof. Let $\mathrm{x} \in \xi_{\gamma} \mathrm{Cl}(\mathrm{A})$ and suppose that $\mathrm{A} \cap \mathrm{V}=\phi$, for some $\xi_{\gamma}$-open set $\mathrm{V}$ of $\mathrm{X}$ containing $\mathrm{x}$. Then, $(\mathrm{X} \backslash \mathrm{V})$ is $\xi_{\gamma}$-closed and $\mathrm{A} \subseteq(\mathrm{X} \backslash \mathrm{V})$, thus $\xi_{\gamma} \mathrm{Cl}(\mathrm{A}) \subseteq(\mathrm{X} \backslash \mathrm{V})$. But, this implies that $\mathrm{x} \in(\mathrm{X} \backslash \mathrm{V})$ which is contradiction. Therefore,

$\mathrm{A} \cap \mathrm{V} \neq \phi$.

Conversely, Let $\mathrm{A} \subseteq \mathrm{X}$ and $\mathrm{x} \in \mathrm{X}$ such that for each $\xi_{\gamma}$-open set $\mathrm{V}$ of $\mathrm{X}$ containing $\mathrm{x}, \mathrm{A}$ $\cap \mathrm{V} \neq \phi$. If $\mathrm{x} \notin \xi_{\gamma} \mathrm{CL}(\mathrm{A})$, there exists an $\xi_{\gamma}$-closed set $\mathrm{F}$ such that $\mathrm{A} \subseteq \mathrm{F}$. Then, $(\mathrm{X} \mid \mathrm{F})$ is an $\xi_{\gamma}$-open set with $\mathrm{x} \in(\mathrm{X} \backslash \mathrm{F})$, and thus $(\mathrm{X} \backslash \mathrm{F}) \cap \mathrm{A} \neq \phi$, which is a contradiction.

The proof of the following two results is obvious.

Proposition 4.15 Let $A$ be a subset of a topological space $(X, \tau)$ and $\gamma$ be an $\xi$-operation on $\xi \mathrm{O}(\mathrm{X})$. Then, $\xi_{\gamma} \mathrm{Cl}(\mathrm{A})=\mathrm{A} \cup \xi_{\gamma} \mathrm{D}(\mathrm{A})$.

Proposition 4.16 If $\mathrm{A}$ and $\mathrm{B}$ are subsets of a space $\mathrm{X}$ with $\mathrm{A} \subseteq \mathrm{B}$. Then, $\xi_{\gamma} \mathrm{Cl}(\mathrm{A}) \subseteq$ $\xi_{\gamma} \mathrm{Cl}(\mathrm{B})$.

Definition 4.17 Let A be a subset of a topological space $(X, \tau)$ and $\gamma$ be an operation on $\xi \mathrm{O}(\mathrm{X})$. The union of all $\xi_{\gamma}$-open sets contained in $\mathrm{A}$ is called the $\xi_{\gamma}$-Interior of $\mathrm{A}$ and denoted by $\xi_{\gamma}$-Int(A).

Here, we introduce some properties of $\xi_{\gamma}$-Interior of the sets.

Proposition 4.18 Let $(X, \tau)$ be a topological space and $\gamma$ be an operation on $\xi \mathrm{O}(\mathrm{X})$. For any subsets $\mathrm{A}$ and $\mathrm{B}$ of $\mathrm{X}$, we have the following:

1) $\xi_{\gamma}$-Int(A) is an $\xi_{\gamma}$-open set in $X$.

2) $\mathrm{A}$ is $\xi_{\gamma}$-open if and only if $\mathrm{A}=\xi_{\gamma}-\operatorname{Int}(\mathrm{A})$.

3) $\left.\xi_{\gamma}-\operatorname{Int}\left(\xi_{\gamma}-\operatorname{Int} \mathrm{A}\right)\right)=\xi_{\gamma}-\operatorname{Int}(\mathrm{A})$. 
4) $\xi_{\gamma}-\operatorname{Int}(\phi)=\phi$ and $\xi_{\gamma}-\operatorname{Int}(\mathrm{X})=\mathrm{X}$.

5) $\xi_{\gamma}-\operatorname{Int}(\mathrm{A}) \subseteq \mathrm{A}$.

6) If $\mathrm{A} \subseteq \mathrm{B}$, then $\xi_{\gamma}-\operatorname{Int}(\mathrm{A}) \subseteq \xi_{\gamma}-\operatorname{Int}(\mathrm{B})$.

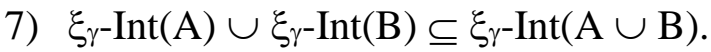

8) $\xi_{\gamma}-\operatorname{Int}(\mathrm{A} \cap \mathrm{B}) \subseteq \xi_{\gamma}-\operatorname{Int}(\mathrm{A}) \cap \xi_{\gamma}-\operatorname{Int}(\mathrm{B})$.

Proof. Straightforward.

In general, the equalities of (7) and (8) of the above proposition do not hold, as is shown in the following examples:

Example 4.19 Consider $X=\{a, b, c, d\}$ with the topology $\tau=\{\phi, X,\{a\},\{b\},\{c\},\{a$, $b\},\{a, c\},\{b, c\},\{a, b, c\}\}$. Define an $\xi$-operation $\gamma$ by.

$$
\gamma(A)= \begin{cases}A & \text { if } b \in A \\ X & \text { if } b \notin A\end{cases}
$$

It is clear that $\xi \gamma-O(X)=\{\phi, X,\{b\},\{a, b\},\{b, c\},\{a, b, c\}\}$. Now, if we let $A=\{a\}$ and $B=\{b\}$, then $\xi \gamma-\operatorname{Int}(A)=\phi$ and $\xi \gamma-\operatorname{Int}(B)=\{b\}$, hence $\xi \gamma-\operatorname{Int}(A) \cup \xi \gamma-\operatorname{Int}(B)=\{b\}$, but $\xi \gamma$-int $(A \cup B)=\{a, b\}$, where $A \cup B=\{a, b\}$, this implies that $\xi \gamma-\operatorname{Int}(A \cup B) \neq \xi \gamma$ $\operatorname{Int}(\mathrm{A}) \cup \xi \gamma-\operatorname{Int}(\mathrm{B})$.

Example 4.20 Consider $X=\{a, b, c\}$ with discrete topology on $X$. Define an $\xi$ operation $\gamma$ on $\xi \mathrm{O}(\mathrm{X})$ by

$$
\gamma(A)=\left\{\begin{array}{lll}
A & \text { if } & A=\{a, b\} \text { or }\{a, c\} \\
X & & \text { otherwise }
\end{array}\right.
$$

Then, $\xi_{\gamma} \mathrm{O}(\mathrm{X})=\{\phi, \mathrm{X},\{\mathrm{a}, \mathrm{b}\},\{\mathrm{a}, \mathrm{c}\}\}$. Now, if we let $\mathrm{A}=\{\mathrm{a}, \mathrm{b}\}$ and $\mathrm{B}=\{\mathrm{a}, \mathrm{c}\}$, then $\xi_{\gamma^{-}}$ $\operatorname{Int}(\mathrm{A})=\{\mathrm{a}, \mathrm{b}\}$ and $\xi_{\gamma}-\operatorname{Int}(\mathrm{B})=\{\mathrm{a}, \mathrm{c}\}$, therefore $\xi_{\gamma}-\operatorname{Int}(\mathrm{A}) \cap \xi_{\gamma}-\operatorname{Int}(\mathrm{B})=\{\mathrm{a}\}$, but $\xi_{\gamma}-\operatorname{Int}(\mathrm{A}$ $\cap \mathrm{B})=\phi$, where $\mathrm{A} \cap \mathrm{B}=\{\mathrm{a}\}$, this implies that $\xi_{\gamma}-\operatorname{Int}(\mathrm{A}) \cap \xi_{\gamma}-\operatorname{Int}(\mathrm{B}) \neq \xi_{\gamma}-\operatorname{Int}(\mathrm{A} \cap \mathrm{B})$.

The following two results can be easily proved.

Proposition 4.21 For any subset A of a topological space $X, \xi_{\gamma}-\operatorname{Int}(\mathrm{A}) \subseteq \xi \operatorname{Int}(\mathrm{A}) \subseteq$ Int(A).

Proposition 4.22 Let $A$ be any subset of a topological space $X$, and $\gamma$ be an operation on $\xi \mathrm{O}(\mathrm{X})$. Then, $\xi_{\gamma}-\operatorname{Int}(\mathrm{A})=\mathrm{A} \backslash \xi_{\gamma}-\mathrm{D}(\mathrm{X} \backslash \mathrm{A})$. 


\section{REFERENCES}

[1] Basu, C. K., Afsan, B. M. U. and Ghosh, M. K., (2009), A class of functions and separation axioms with respect to an operation. Hacettepe journal of Mathematics and Statistics, 38 (2), 103-118.

[2] Dugundji, J., (1966), Topology, Allyn and Bacon Inc., Boston, .

[3] El-Deeb, S. N., Hasanein, I. A., Mashhour, A. S. and Noiri, T., (1983), On Pregular spaces, Ball. Math. Sci. Math. R. S. Rounmanie, 27 (4), 311-315.

[4] Hasan M. Haji, (2010), On Some Types of Continuity, Separation Axioms and Dimension Functions, ph.D. Thesis, Duhok Univ., Duhok, .

[5] Kasahara, S., (1979), Operation compact spaces, Math. Japonica, 24 (1), 97105.

[6] Krishnan, G. S. and Balachandran, K., (2006), On $\gamma$-semi-open sets in topological space, Bull. Cal. Math. Soc., 98 (6), 517-530.

[7] Liven, N., (1963), Semi-open sets and semi-continuity in topological spaces, Amer. Math. Monthly, 70 (1), 36-41.

[8] Moiz K., (1997), Weak forms of continuity, compactness and connectedness, ph.D. Thesis, Multan Univ., Pakistan,.

[9] Ogata, H., (1991), Operation on topological spaces and associated topology, Math. Japonica, 36 (1), 175-184.

[10] Reilly, I. L. and Vamanmurthy, M.K., (1985), On $\alpha$-contiuity in topological spaces, Acta Math. Hungar., 45 (1-2) , 27-32.

[11] Sia sundra Krishnan, G., (2003), A new class of semi-open sets in a topological space, Proc. NCMCM, Allied Publishers, new Delhi, 305-311. 